\title{
ON GREEN'S FUNCTIONS IN THE THEORY OF HEAT CONDUCTION IN SPHERICAL COORDINATES $\dagger$
}

\author{
ARNOLD N. LOWAN
}

In a previous paper, $\ddagger$ the writer derived the expressions for the Green's functions in the theory of heat conduction for an infinite cylinder and for an infinite solid, bounded internally by a cylinder.

The object of the present paper is to derive the appropriate Green's functions for a sphere and for an infinite solid bounded internally by a sphere. In both cases, we shall take the boundary condition in the form

$$
\frac{\partial u}{\partial r}+h u=0, \quad r=a .
$$

The case of a sphere. In this case we start with the expression

$$
u\left(r, \theta, \phi, t ; r_{0}, \theta_{0}, \phi_{0}\right)=\frac{1}{2(\pi k t)^{3 / 2}} e^{-R^{2} / 4 k t},
$$

where

$$
R^{2}=r^{2}+r_{0}^{2}-2 r_{0} \cos \gamma,
$$

$\gamma$ being the angle between the radii from the origin to the points $(r, \theta, \phi)$ and $\left(r_{0}, \theta_{0}, \phi_{0}\right)$. The expression (1) is the point source solution of the differential equation of heat conduction in spherical coordinates.

The expression (1) may be written in the form $\S$

$$
\begin{aligned}
u\left(r, \theta, \phi, t ; r_{0}, \theta_{0} ; \phi_{0}\right)=\frac{1}{4 \pi\left(r r_{0}\right)^{1 / 2}} \sum_{n=0}^{\infty}(2 n+1) P_{n}(\cos \gamma) \\
\cdot \int_{0}^{\infty} \alpha e^{-k \alpha^{2} t} J_{n+1 / 2}\left(\alpha r_{0}\right) J_{n+1 / 2}(\alpha r) d \alpha .
\end{aligned}
$$

The corresponding Laplace transform

$$
L\{u(t)\}=\int_{0}^{\infty} e^{-p t} u(t) d t=u^{*}(p)
$$

$\dagger$ Presented to the Society, October 29, 1938.

$\ddagger$ This Bulletin, vol. 44 (1938), pp. 125-133. This paper will be referred to as A.N.L.

§ See Carslaw, Mathematical Theory of Heat Conduction, article 93. 
is therefore

$$
u^{*}\left(r, \theta, \phi, p ; r_{0}, \theta_{0}, \phi_{0}\right)=\frac{1}{4 \pi\left(r r_{0}\right)^{1 / 2}} \sum_{n=0}^{\infty}(2 n+1) P_{n}(\cos \gamma)
$$

$$
\int_{0}^{\infty} \frac{\alpha d \alpha}{\alpha^{2}-q^{2}} J_{n+1 / 2}(\alpha r) J_{n+1 / 2}\left(\alpha r_{0}\right)
$$

where we have put $p=-k q^{2}$.

With the aid of the identities (5) and $\left(5^{\prime}\right)$ of A.N.L., (4) becomes

(5) $u^{*}=\frac{i}{8 k\left(r r_{0}\right)^{1 / 2}} \sum_{n=0}^{\infty}(2 n+1) P_{n}(\cos \gamma) J_{n+1 / 2}(r q) H_{n+1 / 2}^{1}\left(r_{0} q\right), \quad r<r_{0}$,

(6) $u^{*}=\frac{i}{8 k\left(r r_{0}\right)^{1 / 2}} \sum_{n=0}^{\infty}(2 n+1) P_{n}(\cos \gamma) J_{n+1 / 2}\left(r_{0} q\right) H_{n+1 / 2}^{1}(r q), \quad r>r_{0}$.

In order to obtain the Green's function, we must add to the point source solution $u$ a function $v$, satisfying the differential equation of heat conduction, vanishing at $t=0$, and such that $u+v$ satisfies the boundary condition $\partial u / \partial r+h u=0$, for $r=a$.

Since $L\{\partial u(t) / \partial t\}=L\{u(t)\}-u(0)=u^{*}(p)-u(0)$, and since the two operations of differentiation with respect to $x$, and of acting with the Laplace operator $L$, may be commuted, the Laplace transform of $v$ must satisfy the differential equation

$$
\Delta v^{*}+q^{2} v^{*}=0
$$

The transition from $u^{*}+v^{*}$ to the desired Green's function $G=u+v$. will be apparent from the subsequent developments.

The most general solution of (7) which is symmetric about the axis $\gamma=0$ may be written in the form

$$
v^{*}=\frac{i}{8 k\left(r r_{0}\right)^{1 / 2}} \sum_{n=0}^{\infty}(2 n+1) A_{n} P_{n}(\cos \gamma) J_{n+1 / 2}(r q)
$$

From (8) we get

$$
\begin{aligned}
\left(\frac{\partial v^{*}}{\partial r}+h v^{*}\right)_{r=a}= & \frac{i}{8 k\left(a r_{0}\right)^{1 / 2}} \sum_{n=0}^{\infty}(2 n+1) A_{n} P_{n}(\cos \gamma) \\
& \cdot\left\{q \frac{d}{d z} J_{n+1 / 2}(z)+\left[h-\frac{1}{2 a}\right] J_{n+1 / 2}(z)\right\}_{z=a q} .
\end{aligned}
$$

Since

$$
\left(\frac{\partial}{\partial r}+h\right)\left(u^{*}+v^{*}\right)=0, \quad r=a,
$$


it follows that

(11) $A_{n}=-J_{n+1 / 2}\left(r_{0} q\right) \frac{\left\{q \frac{d}{d z} H_{n+1 / 2}^{1}(z)+(h-1 /(2 a)) H_{n+1 / 2}^{1}(z)\right\}_{z=a q}}{q J_{n+1 / 2}^{\prime}(a q)+(h-1 /(2 a)) J_{n+1 / 2}(a q)} ;$

therefore $\dagger$

$$
u^{*}+v^{*}=\frac{i}{8 k\left(r r_{0}\right)^{1 / 2}} \sum_{n=0}^{\infty}(2 n+1) P_{n}(\cos \gamma) w_{n}^{*},
$$

where

and

$$
\begin{aligned}
w_{n}^{*}=\frac{J_{n+1 / 2}(r q)}{U_{n+1 / 2}(a q)}\left\{H_{n+1 / 2}^{1}(r q) U_{n+1 / 2}(a q)-J_{n+1 / 2}(r q)\right. \\
\left.\cdot\left[\frac{z}{a} \frac{d}{d z} H_{n+1 / 2}^{1}(z)+\left(h-\frac{1}{2 a}\right) H_{n+1 / 2}^{1}(z)\right]_{z=a q}\right\}
\end{aligned}
$$

$$
U_{n+1 / 2}(a q)=q J_{n+1 / 2}^{\prime}(a q)+\left(h-\frac{1}{2 a}\right) J_{n+1 / 2}(a q) .
$$

Comparison between (14) and equation (14) of A.N.L. shows clearly that there is a formal analogy between the present and the former expression for $w_{n}^{*}$. Specifically, our present $w_{n}^{*}$ may be obtained from the corresponding expression in A.N.L. by replacing $n$ by $n+1 / 2$ and $h$ by $h-1 /(2 a)$ and multiplying by the factor $1 / 2$. The inversion of (12) therefore ultimately yields $\ddagger$

$$
\begin{aligned}
& G(r, \theta, \phi, t\left.; r_{0}, \theta_{0}, \phi_{0}\right)=\frac{1}{2 \pi a^{2}\left(r r_{0}\right)^{1 / 2}} \sum_{n=0}^{\infty}(2 n+1) P_{n}(\cos \gamma) \\
& \cdot \sum_{q_{i}} q_{i}{ }^{2} e^{-k q_{i}{ }^{2} t} J_{n+1 / 2}\left(q_{i} r\right) \\
& \cdot \frac{J_{n+1 / 2}\left(q_{i} r_{0}\right)}{\left[(h-1 /(2 a))^{2}+q_{i}{ }^{2}-(n+1 / 2)^{2} / a^{2}\right]\left[J_{n+1 / 2}\left(q_{i} a\right)\right]^{2}},
\end{aligned}
$$

$\dagger$ Formulas (13), (15), (18), (19), (20), and (22) are given for $r<r_{0}$. In the case $r>r_{0}$, the corresponding formulas are obtained by interchanging $r$ and $r_{0}$.

$\ddagger$ As mentioned in A.N.L., the transition from $p w_{n}^{*}=Y(p) / Z(p)$ to $w_{n}$ is equivalent to the inversion of the Laplace transform defining $w_{n}{ }^{*}$, and we have

$$
w_{n}=\frac{Y(0)}{Z(0)}+\sum \frac{Y\left(p_{i}\right)}{p_{i} Z^{\prime}\left(p_{i}\right)} \cdot e^{p_{i} t}
$$

where the summation extends over the roots of $Z(p)=0$. 
where the second summation extends over the roots

$$
U_{n+1 / 2}(a q)=0 .
$$

From this formula we may obtain the Green's function for the case where the boundary is impervious to heat by putting $h=0$. Also the case where the boundary is kept at $0^{\circ}$ may be obtained by putting $h=\infty$. In this case it is clear that the transcendental equation (16) reduces to

$$
J_{n+1 / 2}(a q)=0 .
$$

Also it is easily seen that the denominator of (15) reduces to

$$
q_{i}^{2}\left[J_{n+1 / 2}^{\prime}\left(q_{i} a\right)\right]^{2} .
$$

Thus the Green's function for the case where the boundary is kept at $0^{\circ}$ is

$$
\begin{array}{r}
G\left(r, \theta, \phi, t ; r_{0}, \theta_{0}, \phi_{0}\right)=\frac{1}{2 \pi a^{2}\left(r r_{0}\right)^{1 / 2}} \sum_{n=0}^{\infty}(2 n+1) P_{n}(\cos \gamma) \sum_{q_{i}} e^{-k q_{i}{ }^{2} t} \\
\frac{J_{n+1 / 2}\left(q_{i} r\right) J_{n+1 / 2}\left(q_{i} r_{0}\right)}{\left\{J_{n+1 / 2}^{\prime}\left(a q_{i}\right)\right\}^{2}}
\end{array}
$$

where the second summation extends over the roots of (17).

Case of the infinite solid bounded internally by a sphere. The former analogy with the treatment in A.N.L., noticed in the previous case, applies also in the case under consideration. Thus since $v^{*}$ must be finite for $r=\infty$, it follows that in (8) we must replace $J_{n+1 / 2}(r q)$ by $H_{n+1 / 2}^{1}(r q)$. Proceeding as in the previous case, we ultimately obtain

$$
u^{*}+v^{*}=\frac{i}{8 k\left(r r_{0}\right)^{1 / 2}} \sum_{n=0}^{\infty}(2 n+1) P_{n}(\cos \gamma) W_{n}^{*}
$$

where the expression for $W_{n}^{*}$ may be obtained from equation (30) of A.N.L. by replacing $h$ by $h-1 /(2 a)$ and $n$ by $n+1 / 2$ and multiplying by the factor $1 / 2$. Our final solution is therefore

$$
\begin{aligned}
G\left(r, \theta, \phi, t ; r_{0}, \theta_{0}, \phi_{0}\right)=\frac{1}{8 \pi\left(r r_{0}\right)^{1 / 2}} \sum_{n=0}^{\infty}(2 n+1) P_{n}(\cos \gamma) \\
\cdot \int_{-\infty}^{+\infty} \alpha e^{-k \alpha^{2} t} \frac{H_{n+1 / 2}^{1}\left(\alpha r_{0}\right)}{U_{n+1 / 2}(\alpha a)} \\
\cdot\left\{J_{n+1 / 2}(\alpha r) U_{n+1 / 2}(\alpha a)-U_{n+1 / 2}(\alpha r) J_{n+1 / 2}(\alpha a)\right\} d_{\alpha}
\end{aligned}
$$


where

$$
U_{n+1 / 2}(\alpha a)=\left\{\alpha \frac{d}{d z} H_{n+1 / 2}^{1}(z)+\left(h-\frac{1}{2 a}\right) H_{n+1 / 2}^{1}(z)\right\}_{z=a \alpha} .
$$

For $h=\infty$ this reduces to

$$
\begin{aligned}
G\left(r, \theta, \phi, t ; r_{0}, \theta_{0},\right. & \left.\phi_{0}\right)=\frac{1}{8 \pi\left(r r_{0}\right)^{1 / 2}} \sum_{n=0}^{\infty}(2 n+1) P_{n}(\cos \gamma) \\
& \cdot \int_{-\infty}^{+\infty} \alpha e^{-k \alpha^{2} t} \frac{H_{n+1 / 2}^{1}\left(\alpha r_{0}\right)}{H_{n+1 / 2}^{1}(\alpha a)} \\
& \cdot\left\{J_{n}(\alpha r) H_{n+1 / 2}^{1}(\alpha a)-J_{n+1 / 2}(\alpha a) H_{n+1 / 2}^{1}(\alpha r)\right\} d \alpha .
\end{aligned}
$$

This is the solution of our problem when the spherical surface $r=a$ is kept at $0^{\circ}$.

The Green's functions above evaluated may be called point source Green's functions. They are solutions of the differential equation of heat conduction, depending on the spherical coordinates $r, \theta$, and $\phi$ and satisfying the condition

$$
\lim _{\epsilon \rightarrow 0} \iiint_{\omega} G\left(r, \theta, \phi, 0 ; r^{\prime}, \theta^{\prime}, \phi^{\prime}\right) d \tau=1,
$$

where $\omega$ is a little sphere of radius $\epsilon$ surrounding the point source $\left(r_{0}, \theta_{0}, \phi_{0}\right)$.

In addition to these Green's functions we may consider the Green's functions depending on $r$ only and satisfying the condition

$$
\lim _{\epsilon \rightarrow 0} 4 \pi \int_{r_{0}}^{r_{0}+\epsilon} G(r, \rho, 0) \rho^{2} d \rho=1 .
$$

For the case of the sphere radiating into a medium at $0^{\circ}$, the Green's function, while not given explicitly by Carslaw, may be derived from his article 65 , in the form

$$
\begin{aligned}
G\left(r, t ; r_{0}\right)=\frac{1}{2 \pi a r r_{0}} \sum_{n=1}^{\infty} \frac{a^{2} \alpha_{n}{ }^{2}+(a h-1)^{2}}{a^{2} \alpha_{n}{ }^{2}+a h(a h-1)} \\
\cdot \sin \alpha_{n} r \cdot \sin \alpha_{n} r_{0} e^{-k \alpha_{n}{ }^{2} t},
\end{aligned}
$$

where $\alpha_{n}$ is a root of $a \alpha \cos a \alpha+(a h-1) \sin a \alpha=0$.

The Green's function for the case of the infinite solid bounded internally by a sphere may be obtained by considering a continuous distribution of point sources over the sphere $r=r_{0}$ and integrating for the variables $\theta^{\prime}$ and $\phi^{\prime}$. This leads to 


$$
\begin{aligned}
G\left(r, t ; r_{0}\right)=\frac{1}{2 \pi a^{2}} & \sum_{q_{i}} P_{0}(\cos \gamma) e^{-k q_{i}{ }^{2} t} \\
& \cdot \frac{J_{1 / 2}\left(q_{i} r\right) J_{1 / 2}\left(q_{i} r_{0}\right)}{\left[(h-1 /(2 a))^{2}+q_{i}{ }^{2}-1 /\left(4 a^{2}\right)\right]\left[J_{1 / 2}\left(q_{i} a\right)\right]^{2}},
\end{aligned}
$$

where the summation extends over the roots of (17).

The desired results may also be obtained in the following manner. It can be easily shown that if $u(r, \rho, t)$, is the Green's function appropriate to a "plane source," and therefore satisfying the condition

$$
\lim _{\epsilon \rightarrow 0} \int_{r_{0}}^{r_{0}+\epsilon} u(r, \rho, 0) d \rho=1 \text {, }
$$

then

$$
v=\frac{1}{4 \pi r r_{0}} u
$$

is the desired Green's function appropriate to a spherical source. By substituting for $u$ the expression which may be derived from Carslaw's article 82, the desired Green's function is obtained in the form

$$
\begin{aligned}
& G\left(r, t ; r_{0}\right)=\frac{1}{8 \pi r r_{0}(\pi k t)^{1 / 2}}\left\{\exp \left[-\frac{\left(r-a-r_{0}\right)^{2}}{4 k t}\right]\right. \\
& +\exp \left[-\frac{\left(r-a+r_{0}\right)^{2}}{4 k t}\right] \\
& \left.-2 h \int_{0}^{\infty} e^{-h \xi} \exp \left[-\frac{\left(r-a+r_{0}+\xi\right)^{2}}{4 k t}\right] d \xi\right\}
\end{aligned}
$$

which must, of course, agree with (26).

It should be remarked that the Green's functions so derived are of the general form

$$
G=\sum u_{n}(P) \cdot u_{n}\left(P_{0}\right) e^{-k \lambda n^{2} t},
$$

where the $u_{n}$ 's are the normalized characteristic solutions of the homogeneous differential equation of

$$
\nabla^{2} u+\lambda^{2} u=0
$$

which satisfies the prescribed boundary conditions.

Brooklyn College and

Yeshiva College 